NASA Technical Memorandum 101950

AIAA-89-0970

\title{
Large Amplitude Acoustic Excitation of Swirling Turbulent Jets
}

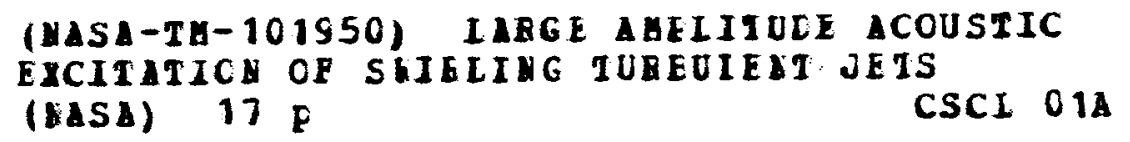

R. Taghavi

Center for Research Inc.

The University of Kansas

Lawrence, Kansas

\section{E.J. Rice}

Lewis Research Center

Cleveland, Ohio

\section{S. Farokhi}

The University of Kansas

Lawrence, Kansas

Prepared for the

2nd Shear Flow Conference

sponsored by the American Institute of Aeronautics and Astronautics

Tempe, Arizona, March 13-16, 1989

\section{N/SA}


LARGE AMPLITUDE ACOUSTIC EXCITATION OF SWIRLING TURBULENT JETS

\author{
R. Taghavi \\ Center for Research Inc. \\ The University of Kansas \\ Lawrence, Kansas \\ E.J. Rice \\ National Aeronautics and Space Administration \\ Lewis Research Center \\ Cleveland, Ohio 44135 \\ and \\ S. Farokhi \\ Aerospace Engineering Department \\ The University of Kansas \\ Lawrence, Kansas
}

\begin{abstract}
SUMMARY
A swirling jet with a swirl number of $S=0.12$ is exited by plane acoustic waves at various Strouhal numbers $\left(S t=f D / U_{\alpha}\right)$. The maximum forcing amplitude of excitation was at 6.88 percent of the time-mean axial velocity at a Strouhal number of $S t=0.39$. The maximum time-mean tangential and axial velocities at the nozzle exit were 18 and $84 \mathrm{~m} / \mathrm{sec}$ respectively. It was observed that the swirling jet was excitable by plane acoustic waves and the "preferred" Strouhal number based on the nozzle diameter and exit axial velocity of the jet was about 0.39 . As a result of excitation at this frequency, the time-mean axial velocity decayed faster along the jet centerline, reaching about 89 percent of its unexcited value at $x / D=9$. Also the half velocity radius and momentum thickness, at 7 nozzle diameters downstream, increased by 13.2 and 5.8 percent respectively, indicating more jet spread and enhanced mixing. To our knowledge, this is the first reported experimental data indicating any mixing enhancement of swirling jets by acoustic excitation.
\end{abstract}

\title{
INTRODUCTION
}

Controlled excitation of nonswirling jets has been extensively studied in the past few years by many investigators including Crow and Champagne (ref. 1), Chan (refs. 2 and 3), Moore (ref. 4), Hussain and Zaman (refs. 5 and 6), and Ahuja et al. (ref. 7). Excitation at the right level and Strouhal number has been shown to result in a faster spread rate due to the higher entrainment caused by the engulfing action of the large-scale coherent structures in the initial region of the mixing layer.

Zaman and Hussain (refs. 8 and 9) showed that turbulence in a circular nonswirling jet is enhanced by excitation at Strouhal numbers between 0.2 and 0.8 and is suppressed between 2 and 4 . They al so concluded that enhancement or suppression of turbulence not only depends on the excitation Strouhal number, but also is affected by the nature of the nozzle boundary layer (i.e., laminar, transitional, or turbulent). Moore (ref. 4) and Ahuja et al. (ref. 7) showed that the threshold level of the acoustic pressure excitation 
for effective turbulent mixing, and a consequential jet noise amplification, can be taken to be 0.08 percent of the jet dynamic pressure at the correct Strouhal number.

Turbulent jets with swirl exhibit distinctive characteristics absent in their nonswirling counterparts. Swirling flows are primarily driven in the nearfield $(x / D<5)$ by the static pressure gradients in both axial and radial directions, consequently the mechanism for jet spread is mainly an inviscid phenomenon. Swirling jets are also inherently highly turbulent. The above characteristics make these flows not easily responsive to any kind of excitation. Mixing enhancement and control of swirling flows are of interest, however, from the point of view of improving the performance of many engine components such as combustion chambers and rotating turbomachinery, as well as vortex-lift devices for external aerodynamics.

The recent study of the controlled excitation of a cold turbulent swirling jet by the authors (refs. 10 to 12), was the first experimental attempt towards basic understanding of this phenomenon. The experiments were conducted for a flow with swirl number of 0.35 (defined as the ratio of the flux of angular momentum to axial momentum normalized by nozzle exit radius). The time-mean axial velocity distribution did not have a "top-hat" radial profile at the nozzle exit. The excitation level of plane acoustic waves was held constant at $124 \mathrm{~dB}$ at various Strouhal numbers. The results showed that even though the axial velocity distribution at the nozzle exit did not have a "top-hat" profile, the instability waves were amplified rapidly in the streamwise direction, reaching a maximum in ampltiude and then decaying further downstream. Excitation at a Strouhal number of 0.4 exhibited the largest growth. Furthermore, it was observed from these results that the instability waves peaked closer to the nozzle exit and their maximum amplitudes were only about 50 percent of their counterparts in the nonswirling jet having the same mass flux, Mach number and Reynolds number.

The objective of the present study was to further investigate the excitability of swirling jets by plane acoustic waves. The emphasis of the research was to study the influence of excitation on mean flow characteristics. To accomplish this, a new acoustic driver system capable of providing much larger excitation amplitudes was used. Also the experiments were conducted at lower swirl numbers than the previous experiments and with radial profiles of axial velocity that were more nearly "top-hat". Effects of swirl number on jet excitability were studied by comparing the response of two jets at different swirl numbers to acoustic excitation. Some comparisons of the results with that of a nonswirling jet generated in the same facility are also made.

\section{NOMENCLATURE}

D

f

$G_{x} \quad$ axial momentum flux, $=2 \pi \int_{0}^{\infty}\left(\rho u^{2}+\left(p-p_{\infty}\right)\right) r d r$ 


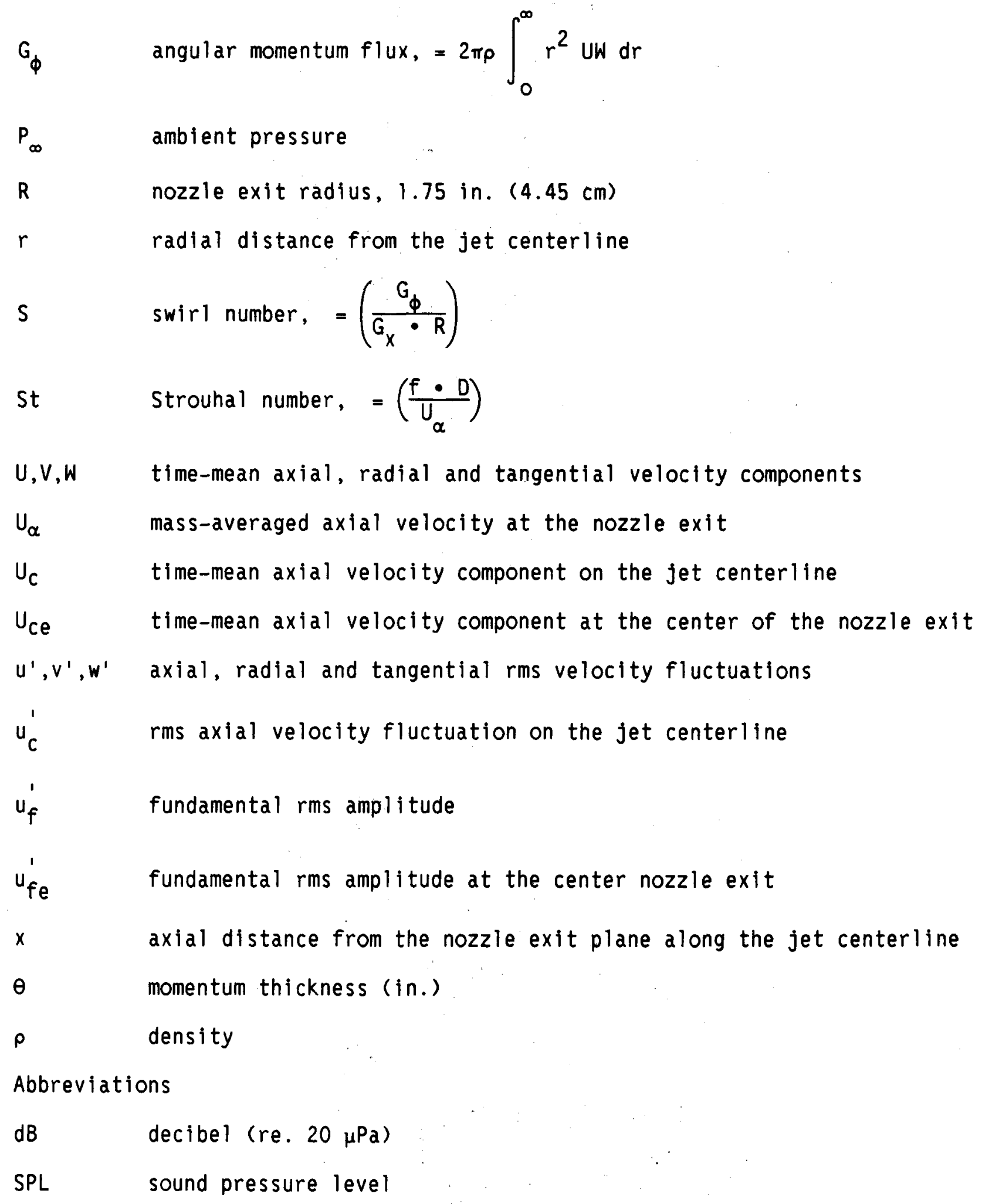




\section{EXPERIMENTAL FACILITY}

\section{Swirl-Generator and Excitation Section}

Figure 1 is a schematic diagram of the test set-up. Plane acoustic waves were generated by two Ling Model EPT-94B Electro-Pneumatic acoustic drivers, positioned $180^{\circ}$ apart around a $16.14 \mathrm{in}$. (41 cm) cylindrical section and operated in phase. Each driver is an electrically-controlled air modulator capable of generating $170 \mathrm{~dB}$ SPL in the near field. The drivers were operated by an air supply of $124 \mathrm{psig}\left(87184 \mathrm{~kg} / \mathrm{m}^{2}\right)$ at a maximum flow rate of $0.61 \mathrm{~b} / \mathrm{sec}$ $(0.27 \mathrm{~kg} / \mathrm{sec})$. The air leaving the drivers was directed in the tangential direction by means of scoops just inside of the plenum. After leaving the plenum, the swirling air was passed through a 30 mesh screen and a trip-ring before entering the $3.5 \mathrm{in}$. $(8.89 \mathrm{~cm})$ diameter nozzle. The screen and tripring were located $13 \mathrm{in}$. $(33 \mathrm{~cm})$ upstream of the nozzle exit where the diameter of the contracting section was $5.16 \mathrm{in.}(13.1 \mathrm{~cm})$. The nozzle had an 8.66 in. $(22 \mathrm{~cm})$ long cylindrical section prior to its exit. The complete jet facility, with different arrangement of acoustical drivers, is shown in figure 2 .

\section{Instrumentation}

Three components of time-mean velocity, as well as static and total pressures were measured by a 5-hole pitot probe having a diameter of $0.125 \mathrm{in}$. $(0.318 \mathrm{~cm})$ at the measuring tip. The probe tip has a $45^{\circ}$ cone angle and the pressure ports are located at the midspan of the conical surface. The 5-hole probe is self-nulling in the yaw direction, while the pitch angle, time-mean velocity components, as well as mean pressures are computed from the measured pressures and the probe calibration curves. For further details, references 10 and 11 can be consulted.

The axial component of fluctuating velocity was measured along the jet centerline using a TSI model 1260A-10 hot wire probe and a DISA model 55 M01 constant temperature anemometer employing a DISA model $55 \mathrm{M} 25$ linearizer. Along the jet axis, the tangential and radial velocity components are negligible compared to the axial component, and therefore the results from a single element hot-wire probe were assumed to represent the actual streamwise velocity fluctuations. The fundamental-rms amplitude at each streamwise location was obtained by analyzing the hot-wire spectra at the excitation frequency. Even though no phase averaging technique was applied, the amplitude of the fundamental wave was significantly above the background noise, which insured negligible contamination of the instability wave amplitude with background turbulent noise. To prevent the probe holder support from entering the flowfield and contaminating the data due to its vibration, only half traverses were made starting from the jet centerline for both hot wire and 5-hole probe measurements.

Excitation sound pressure level and fluctuating pressure spectra, at the center of the nozzle exit, were measured using a model 4135 ( $B$ \& $K$ ) microphone. The microphone has an outside diameter of $0.25 \mathrm{in} .(0.64 \mathrm{~cm})$ and was fitted with a bullet head fairing. The sound pressure level in $\mathrm{dB}$ ( $\mathrm{re} .20 \mu \mathrm{Pa}$ ). at the excitation frequency, was obtained from the spectra of the microphone signal using a Wavetek model $804 \mathrm{~A}$ signal analyzer. 
In this study, the excitability of a swirling jet, with a mass flow rate of $1.2 \mathrm{lb} / \mathrm{sec}(0.54 \mathrm{~kg} / \mathrm{sec})$ was experimentally investigated. The experiments were conducted by exciting a free jet with a swirl number of $S=0.12$ by plane acoustics waves. The swirl number here is defined as

$$
S=\int_{0}^{\infty} \rho U W r^{2} d r / R \int_{0}^{\infty}\left[\rho U^{2}+\left(p-p_{\infty}\right)\right] r d r
$$

The maximum time-mean tangential and axial velocities at the nozzle exit plane were $58.8 \mathrm{fps}(17.9 \mathrm{~m} / \mathrm{sec})$ and $275 \mathrm{fps}(83.8 \mathrm{~m} / \mathrm{sec})$ respectively. The respective Mach and Reynolds numbers of the jet based on the mass averaged axial velocity at the nozzle exit where 0.22 and 460000 . The maximum forcing amplitude of the excitation was 6.88 percent of time-mean axial velocity at a Strouhal number of $S t=0.39$, measured at the center of the nozzle exit plane.

\section{RESULTS}

\section{Mean Flow Measurements}

The radial distributions of time-mean axial velocity at various downstream locations are plotted in figure $3(a)$. The profile has almost a "top hat" distribution at the nozzle exit and transitions to more of a Gaussian shape after about 1.5 nozzle diameters downstream. The time-mean swirl (tangential) velocity has a radial profile at the nozzle exit which resembles that of a Rankine-type vortex, predominated by solid-body rotation, as shown in figure $3(b)$. The downstream decay of the maximum swirl velocity is much faster than the decay of the axial velocity component, as expected.

\section{Instability Wave Measurements}

The jet was excited at various frequencies, by plane acoustic waves. The variation of the rms amplitude of velocity fluctuations at the fundamental excitation frequency $\left(u_{f}^{\prime}\right)$, along the jet centerline, corresponding to various excitation Strouhal numbers, is shown in figure 4. The "preferred" Strouhal number based on the nozzle exit diameter, mass averaged axial velocity, and excitation frequency was about 0.39 as indicated in the figure. The forcing amplitude of the excitation at this frequency was 6.88 percent of the time-mean centerline axial velocity at the nozzle exit $\left(u_{f e}^{\prime} / u_{c e}\right)$. The axial location of the "saturation" point was at $x / D=2$. the growth and decay of the instability wave agrees with the data of Raman et. al (refs. 13 and 14) for highly turbulent nonswirling jets excited at high amplitudes.

Figure 5 shows the streamwise evolution of velocity spectra along the jet axis at an excitation frequency of $330 \mathrm{~Hz}(\mathrm{St}=0.39)$. The isolated peaks at 
$660 \mathrm{~Hz}$ (first harmonic) and $990 \mathrm{~Hz}$ (second harmonic) were not amplified by the flow and therefore not considered in this study. Also from this figure, it is clear that no growth of the subharmonic (165 Hz) is experienced in the case of the swirling jet excited by plane waves. This observation is quite different from that of the nonswirling jets, in which considerable growth of the subharmonic is measurable as a result of excitation at $s t=0.5$ (ref. 15).

Distributions of total axial turbulence intensity along the jet centerline for the unexcited and excited $(S t=0.39$ ) cases are compared in figure $6(a)$. It is seen that as a result of excitation, the total axial turbulence intensity at the nozzle exit has been almost doubled and the location of its maximum value on the jet axis has moved upstream from $x / D=6$ to $x / D=2.5$. For a similar excited jet without swirl, the peak value is reached at a location much further downstream $(x / D=9)$ (ref. 13).

\section{Influence of Excitation on Mean Flow}

The main purpose of exciting the jet is usually to enhance mixing. Alterations in mixing can be observed by comparing the mean flow parameters with and without excitation. The decay of the time-mean axial velocity along the jet axis is compared for excited and unexcited cases in figure $6(b)$. From this figure it is clear that excitation results in a faster decay starting immediately downstream from the nozzle exit. The faster decay of the mean centerline axial velocity is an indication of more jet spreading and enhanced mixing. The enhanced mixing is further confirmed in figure 7 , where the radial distributions of mean axial velocity at $x / d=7$, for excited and unexcited cases are compared. The half velocity radius has increased by about 13.2 percent as a result of excitation.

\section{Comparison of Swirling and Nonswirling Jets}

The variation of momentum thickness along the jet axis for the unexcited swirling jet with swirl number of $S=0.12$ is plotted in figure 8 . The value for the excited swirling jet at $x / D=7$ is also shown, which indicates an increase of about 5.8 percent over the unexcited case and is a further indication of enhanced mixing. The momentum thickness used here is defined as

$$
\theta=\int_{0}^{\infty}\left[\left(U / U_{c}\right) \cdot\left(1-U / U_{c}\right)\right] d r
$$

Data for a similar jet but without swirl (ref. 16) are also plotted in the same figure. For a given axial location, this figure indicates that the momentum thickness for the swirling jet is higher than that of the nonswirling jet.

This is an indication of higher spread rate of the swirling jet compared to the nonswirling jet at all axial locations. It also indicates that the radial gradient of the time-mean axial velocity for the swirling jet is less than the corresponding value of the nonswirling jet. Since, based on the linear stability theory (ref. 17) the growth of the instability wave is proportional to the magnitude of the mean axial velocity gradient, then at a given location along 
the jet centerline, the instability wave has a lower growth rate for the swirling jet as compared to the nonswirling jet under the same conditions. Therefore, the effect of excitation on a swirling jet is less pronounced, compared to the nonswirling jet. This also suggests that, mixing enhancement of swirling jets by excitation requires forcing at higher amplitudes compared to the nonswirling jets.

\section{Swirl Number Variation}

To investigate the effect of swirl number on jet excitability, the 30 mesh screen, which was located upstream of the nozzle was removed. As a result, the maximum time-mean axial and tangential velocities were increased to 286 fps $(87.2 \mathrm{~m} / \mathrm{sec})$ and $86.3 \mathrm{fps}(26.3 \mathrm{~m} / \mathrm{sec})$ respectively as shown in figures $9(\mathrm{a})$ and (b). The swirl number was consequently increased from $S=0.12$ to $S=0.18$. Distributions of time-mean axial velocity and turbulence intensity along the jet axis, for the jets with the two different swirl numbers studied are plotted in figures 10 and 11 . It seems, from figure 10, that with an increase in swirl number and with no excitation, the decay of time-mean axial velocity started further upstream and almost immediately after the nozzle exit, and the "nominal" potential core disappeared. The turbulence intensity at the nozzle exit is also increased for the higher swirl number case by about 60 percent and the downstream location of its maximum value is moved further upstream from $x / D=6$ to about $x / D=3$ ( $f i g .11$ ).

The jet, with the higher swirl number of $S=0.18$, was then excited at the same amplitude as before and at various frequencies. Even though the instability waves exhibited growth along the jet axis (data not shown in this paper), no effect on spread rate and mixing enhancement was observed as a result of excitation. In addition to the issue of decreased radial gradient of axial velocity which was discussed before, another possible explanation for the above can be seen from figure 11. The distribution of turbulence intensity, along the jet axis, for the unexcited jet at the higher swirl number of $S=0.18$ almost coincides with that of the excited jet with $S=0.12$. Therefore the higher swirling jet seems to be self excited and consequently may be insensitive to any additional excitation. The higher initial turbulence of the jet with swirl number of $S=0.18$ compared to the jet at $S=0.12 \mathrm{might}$ also be another explanation for unexcitability of this jet (ref. 15). According to this reference, "increasing the upstream turbulence diminishes the excitability of the jet and reduces the effect of excitation on the spreading rate of the jet."

More parametric studies are scheduled to isolate the other effects such as, maximum tangential velocity at the nozzle exit and excitation level as well as Mach and Reynolds numbers.

\section{CONCLUSIONS}

Plane wave acoustic excitation resulted in a growth and subsequent decay of the shear layer instability wave of a swirling jet having a swirl number of $S=0.12$. This behavior was similar to that of a non-swirling jet. No growth of the subharmonic was observed along the jet axis at the excitation frequencies examined. The "preferred" Strouhal number, based on mass averaged axial velocity and nozzle exit diameter, was about 0.39 . Excitation at this 
frequency resulted in a faster decay of time-mean axial velocity along the jet centerline. The half velocity radius and momentum thickness, at seven nozzle diameters downstream, increased by about 13.2 and 5.8 percent respectively, indicating more jet spreading and mixing enhancement. An increase in swirl number, from $S=0.12$ to $S=0.18$ resulted in drastic changes in the time-mean and fluctuating velocity distributions. The turbulence intensity at the center of the nozzle exit was increased by about 60 percent at the higher swirl number. The higher swirling jet $(S=0.18)$ was also excited at the same amplitudes and various frequencies as before. Even though the growth of the instability wave was measured along the jet axis, no effect on the jet spread rate and mixing enhancement was noticed as a result of excitation. Additional parametric studies designed to further investigate the above phenomenon, will be conducted shortly.

\section{REFERENCES}

1. Crow, S.C. and Champagne, F.H., "Orderly Structure in Jet Turbulence," Journal of Fluid Mechanics, Vol. 48, Part 3, Aug. 16, 1971, pp. 547-591.

2. Chan, Y.Y., "Spatial Waves in Turbulent Jets," Physics of Fluids, Vol. 17, No. 1, Jan. 1974, pp. 46-53.

3. Chan, Y.Y., "Spatial Waves in Turbulent Jets, Part 2," Physics of Fluids, Vol. 17, No. 9, Sept. 1974, pp. 1667-1670.

4. Moore, C.J., "The Role of Shear-Layer Instability Waves in Jet Exhaust Noise," Journal of Fluid Mechanics, Vol. 80, Part 2, Apr. 25, 1977, pp. $321-367$.

5. Hussain, A.K.M.F. and Zaman, K.M.B.Q., "The 'Preferred Mode' of the Axisymmetric Jet," Journal of Fluid Mechanics, Vol. 110, Sept. 1981, pp. 39-71.

6. Hussain, A.K.M.F. and Zaman, K.M.B.Q., "Vortex Pairing in a Circular Jet Under Controlled Excitation. Part 2. Coherent Structure Dynamics," Journal of Fluid Mechanics, Vol. 101, Part 3, Dec. 11, 1980, pp. 493-544.

7. Ahuja, K.K., Lepicovsky, J., Tam, C.K.W., Morris, P.J., and Burrin, R.H., "Tone-Excited Jet: Theory and Experiments," NASA CR-3538, 1982.

8. Zaman, K.M.B.Q. and Hussain, A.K.M.F., "Vortex Pairing in a Circular Jet Under Controlled Excitation. Part I. General Jet Response," Journal of Fluid Mechanics, Vol. 101, Part 3, Dec. 11, 1980, pp. 449-49

9. Zaman, K.M.B.Q. and Hussain, A.K.M.F., "Turbulence Suppression in Free Shear Flows By Controlled Excitation," Journal of Fluid Mechanics, Vol. 103, Feb. 1981, pp. 133-160.

10. Taghavi, R., "Experimental Investigation of Swirling Turbulent Jets," Ph.D. Thesis, The University of Kansas, 1988.

11. Taghavi, R. and Farokhi, S.,"Turbulent Swirling Jets With Excitation," NASA CR-180895, 1988. 
12. Taghavi, R., Rice, E.J., and Farokhi, S., "Controlled Excitation of a Cold Turbulent Swirling Free Jet," Journal of Vibration, Acoustics, Stress and Reliability in Design, Vol. 110, No. 2, Apr. 1988, pp. 234-237.

13. Raman, G., Zaman, K.B.M.Q., and Rice, E.J., "Initial Turbulence Effect on Jet Evolution With and Without Tonal Excitation," AIAA Paper 87-2725, Oct. 1987 (NASA TM-100178).

14. Raman, G., Rice, E.J., and Mankbadi, R.R., "Saturation and the Limit of Jet Mixing Enhancement by Single Frequency Plane Wave Excitation:

Experiment and Theory," First National Fluid Dynamics Congress, Part 2, AIAA, New York, 1988, pp. 1000-1007 (NASA TM-100882).

15. Mankbadi, R., Raman, G., and Rice, E., "Effects of Core Turbulence on Jet Excitability," AIAA Paper 89-0966, Mar. 1989.

16. Raman, G. and Rice, E., "Subharmonic and Fundamental High Amplitude Excitation of an Axisymmetric Jet," AIAA Paper 89-0993, Mar. 1989.

17. Lin, C.C., The Theory of Hydrodynamic Stability, Cambridge University Press, London, 1955.

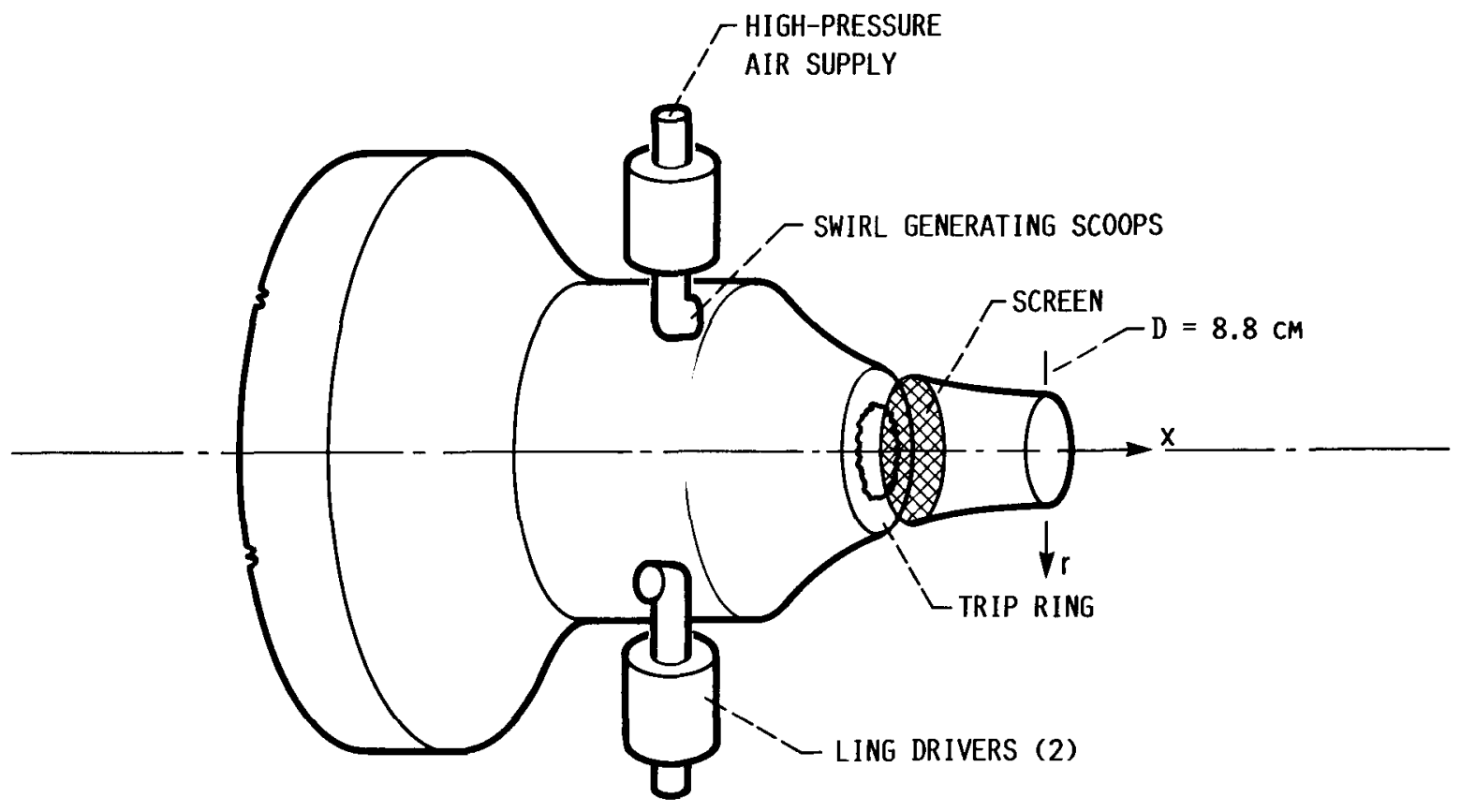

FIGURE 1. - SCHEMATIC OF JET FACILITY. 


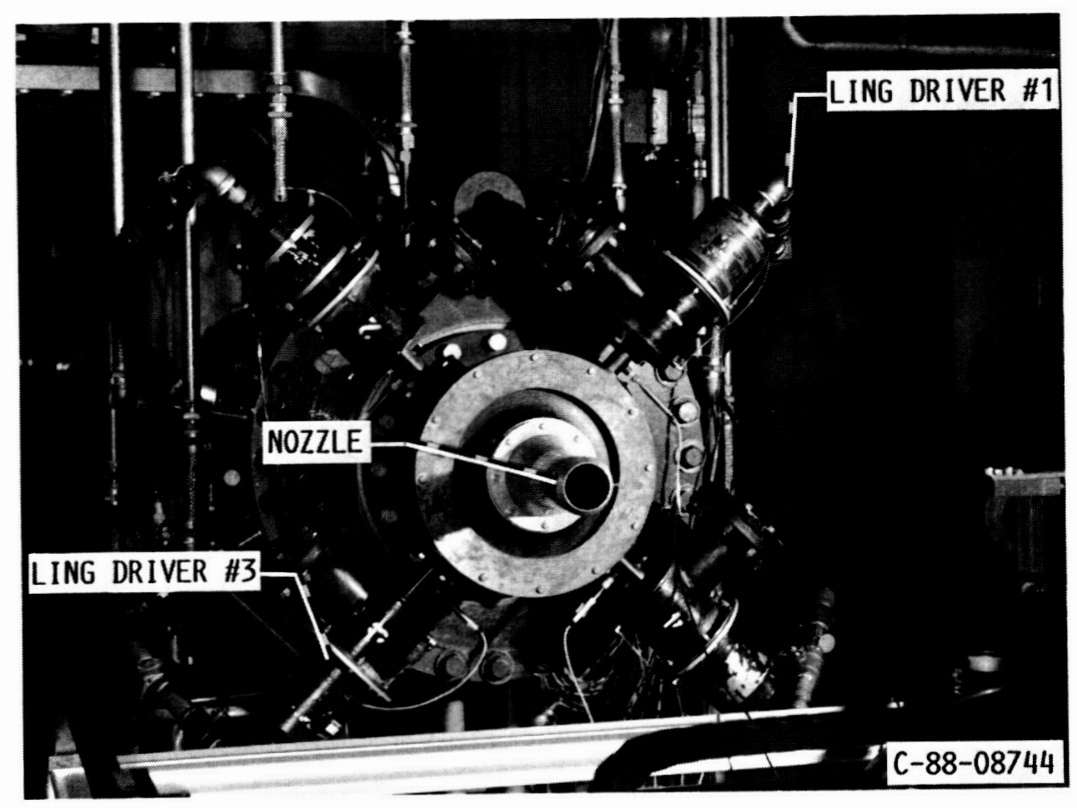

FIGURE 2. - EXPERIMENTAL FACILITY.

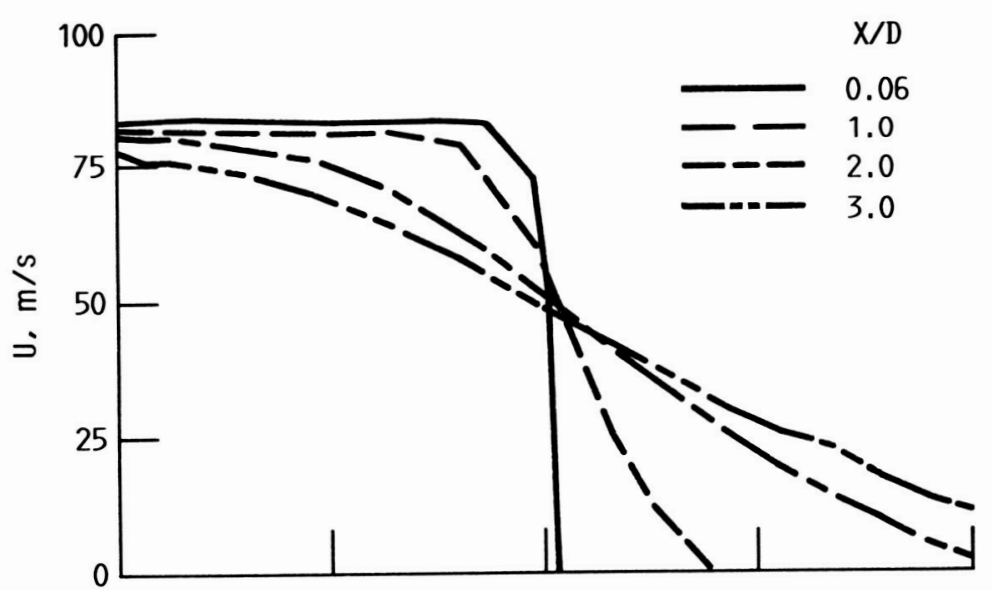

(a) AXIAL.

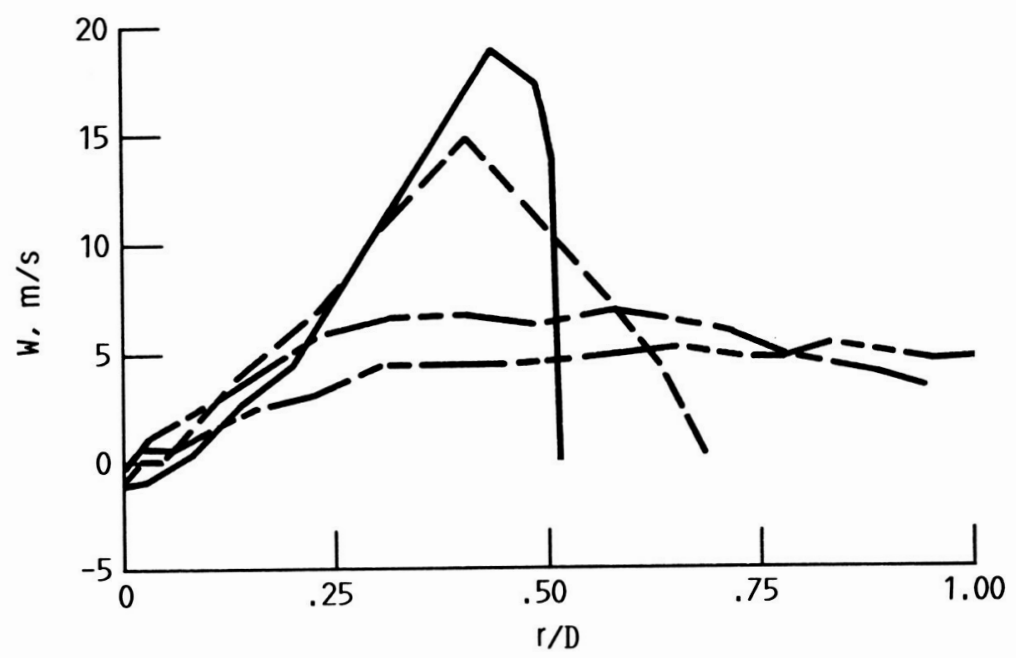

(b) TANGENTIAL.

FIGURE 3. - DOWNSTREAM DEVELOPMENT OF THE TIME-MEAN VELOCITY COMPONENTS. $S=0.12, M=0.22$. 


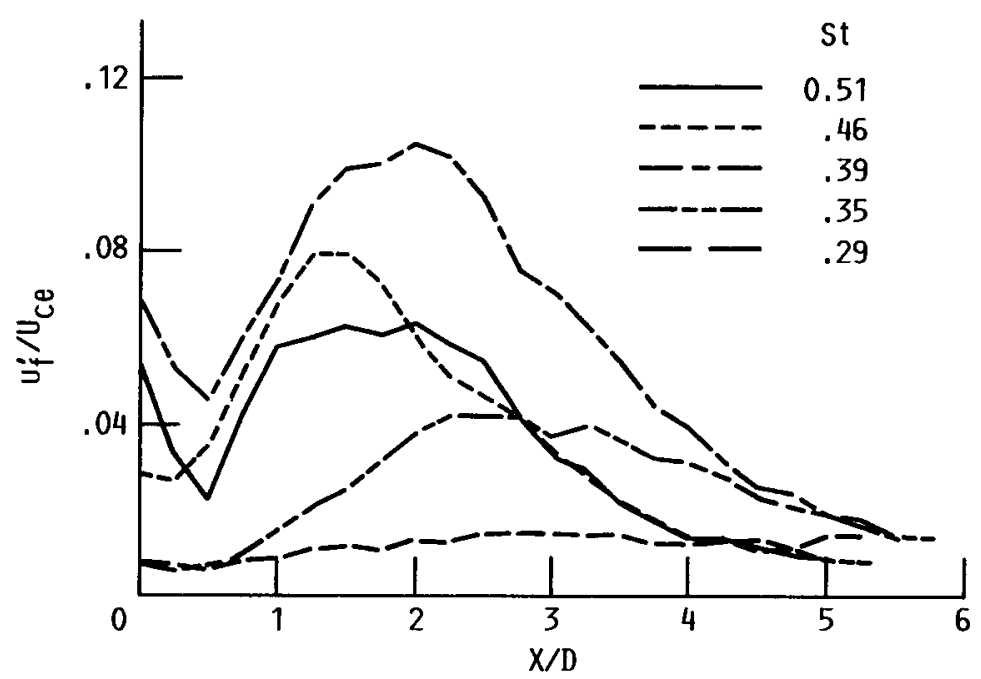

FIGURE 4, - GROWTH OF THE FUNDAMENTAL WAVE ALONG THE JET CENTERLINE. $S=0.12, M=0.22$.

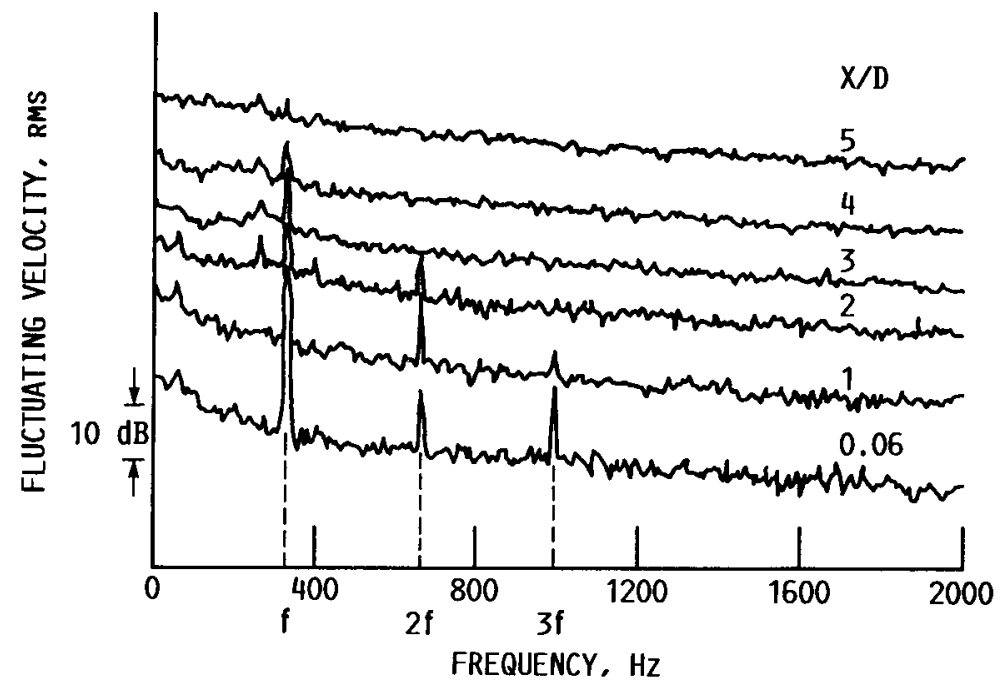

FIGURE 5. - EVOLUTION OF U-SPECTRA ALONG THE JET AXIS, St $=0.39, S=0.12, M=0.22$, BANDWIDTH $=7.5 \mathrm{~Hz}$. 

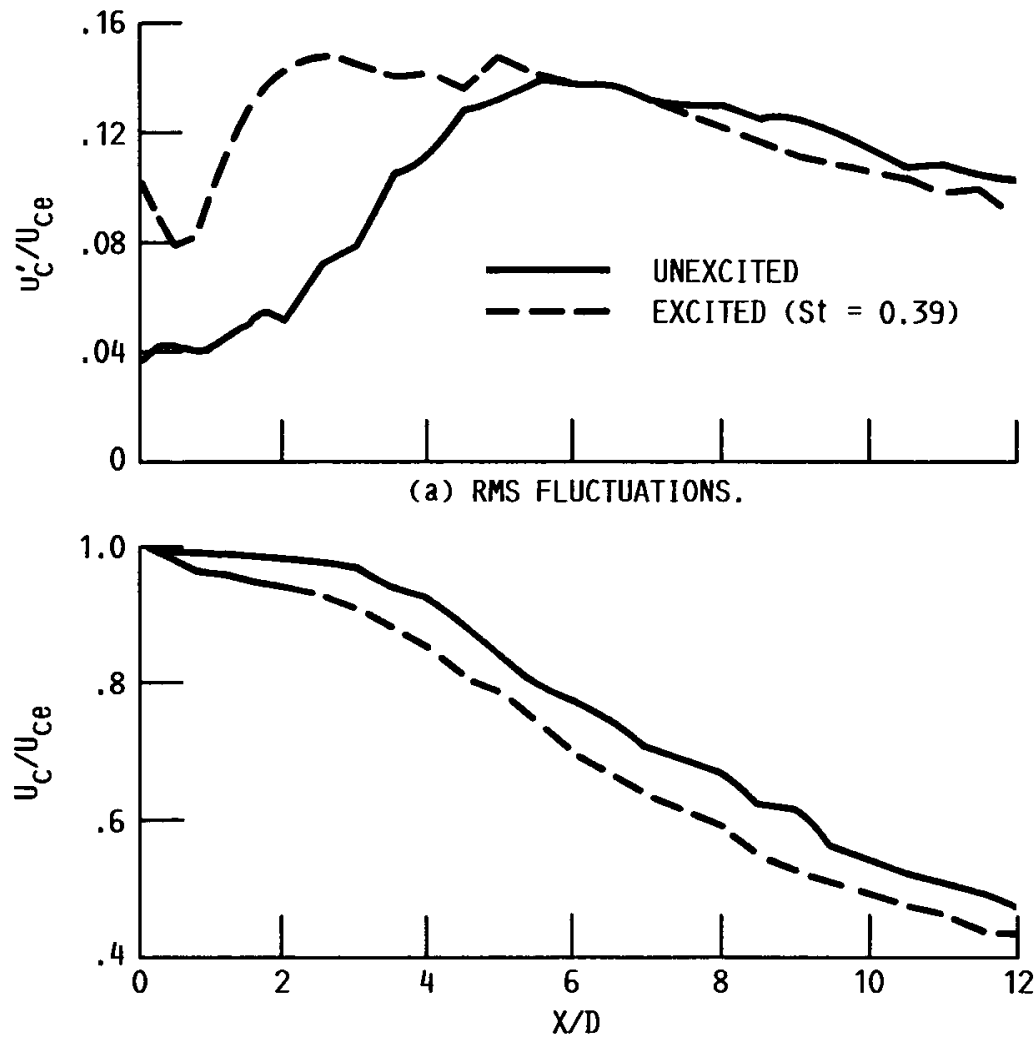

(b) MEAN.

FIGURE 6. - EFFECT OF EXCITATION ON THE AXIAL VELOCITY COMPONENTS ALONG THE JET AXIS. $S=0.12, M=0.22$. 


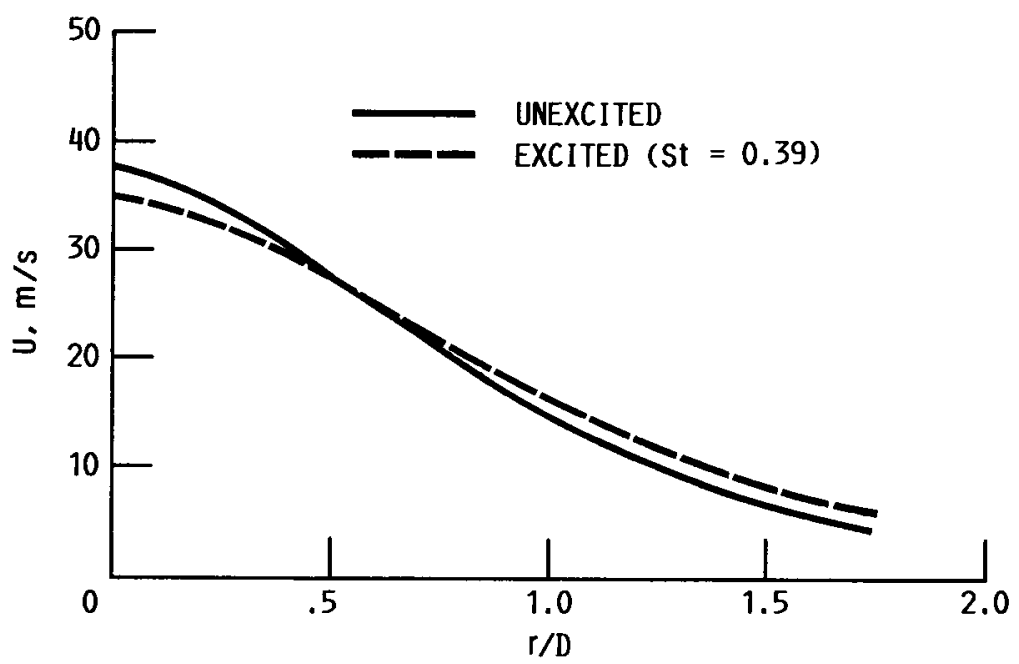

FIGURE 7. - RADIAL DISTRIBUTIONS OF TIME-MEAN AXIAL VELOCITY AT $X / D=7$ WITH AND WITHOUT EXCITATION $(S=0.12)$.

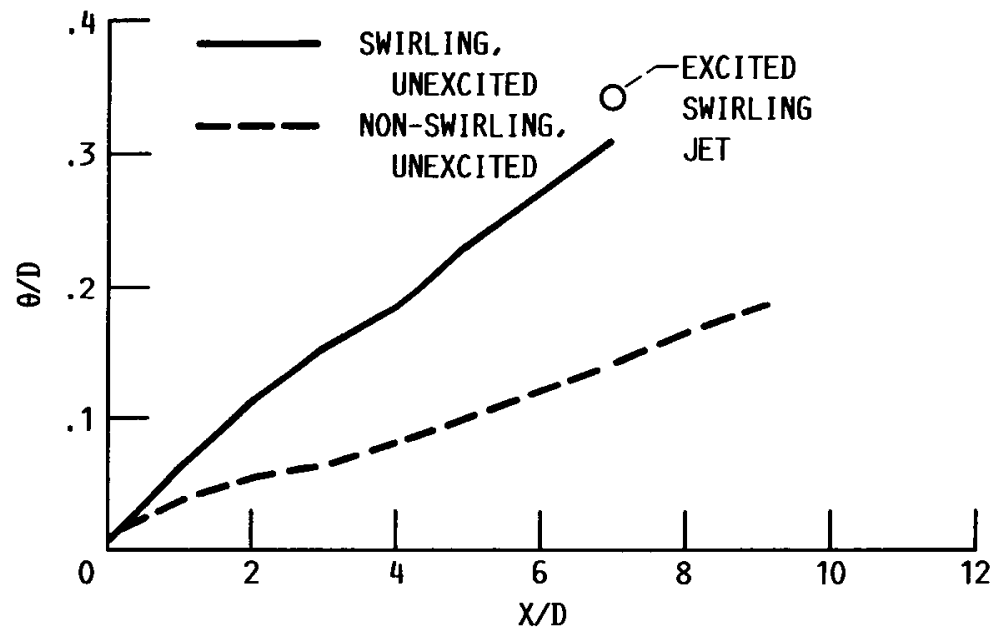

FIGURE 8. - VARIATION OF MOMENTUM THICKNESS ALONG THE JET AXIS. 

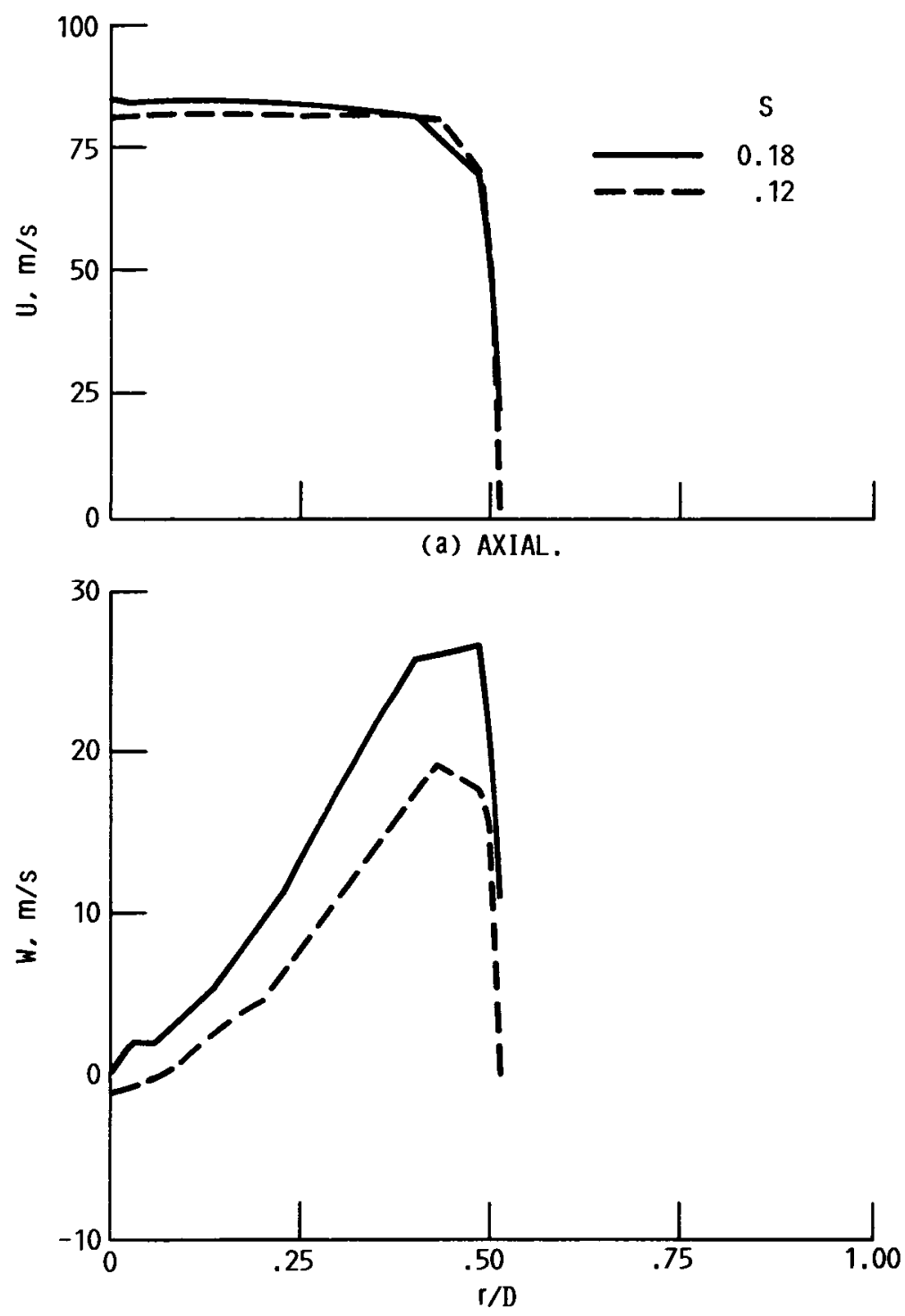

(b) TANGENTIAL.

FIGURE 9. - RADIAL DISTRIBUTIONS OF THE TIME-MEAN VELOCITY COMPONENTS AT THE NOZZLE EXIT AT DIFFERENT SWIRL NUMBERS. 


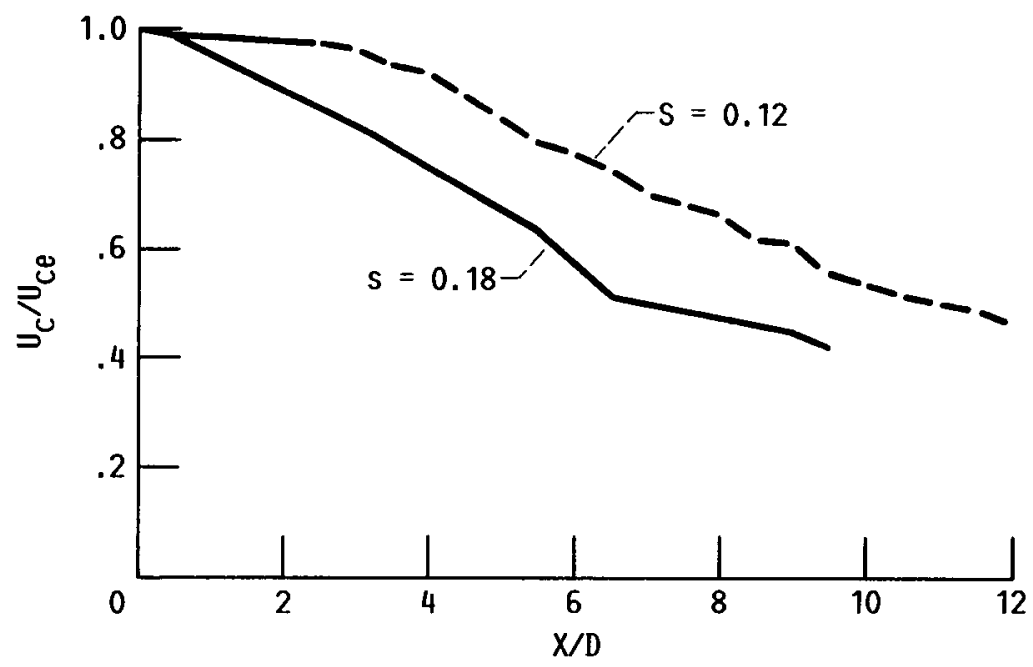

FIGURE 10. - EFFECT OF SWIRL NUMBER ON THE DOWNSTREAM DEVELOPMENT OF THE TIME-MEAN AXIAL VELOCITY ALONG THE JET AXIS. UNEXCITED.

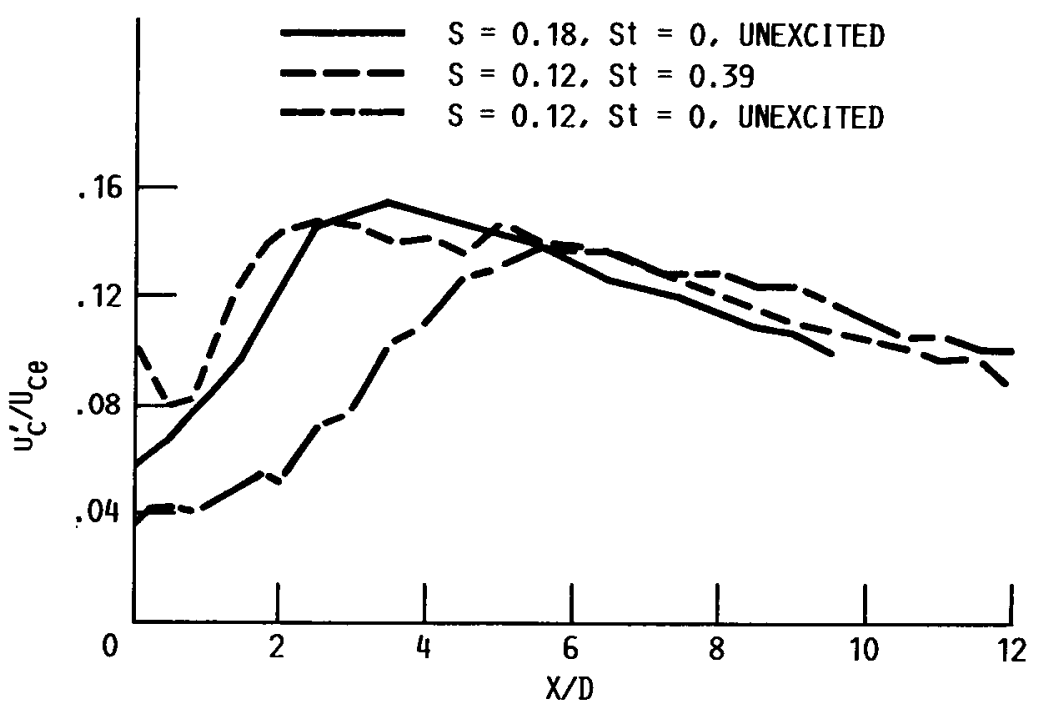

FIGURE 11. - EFFECT OF SWIRL NUMBER AND EXCITATION ON THE DISTRIBUTION TURBULENCE INTENSITY ALONG THE JET AXIS. 


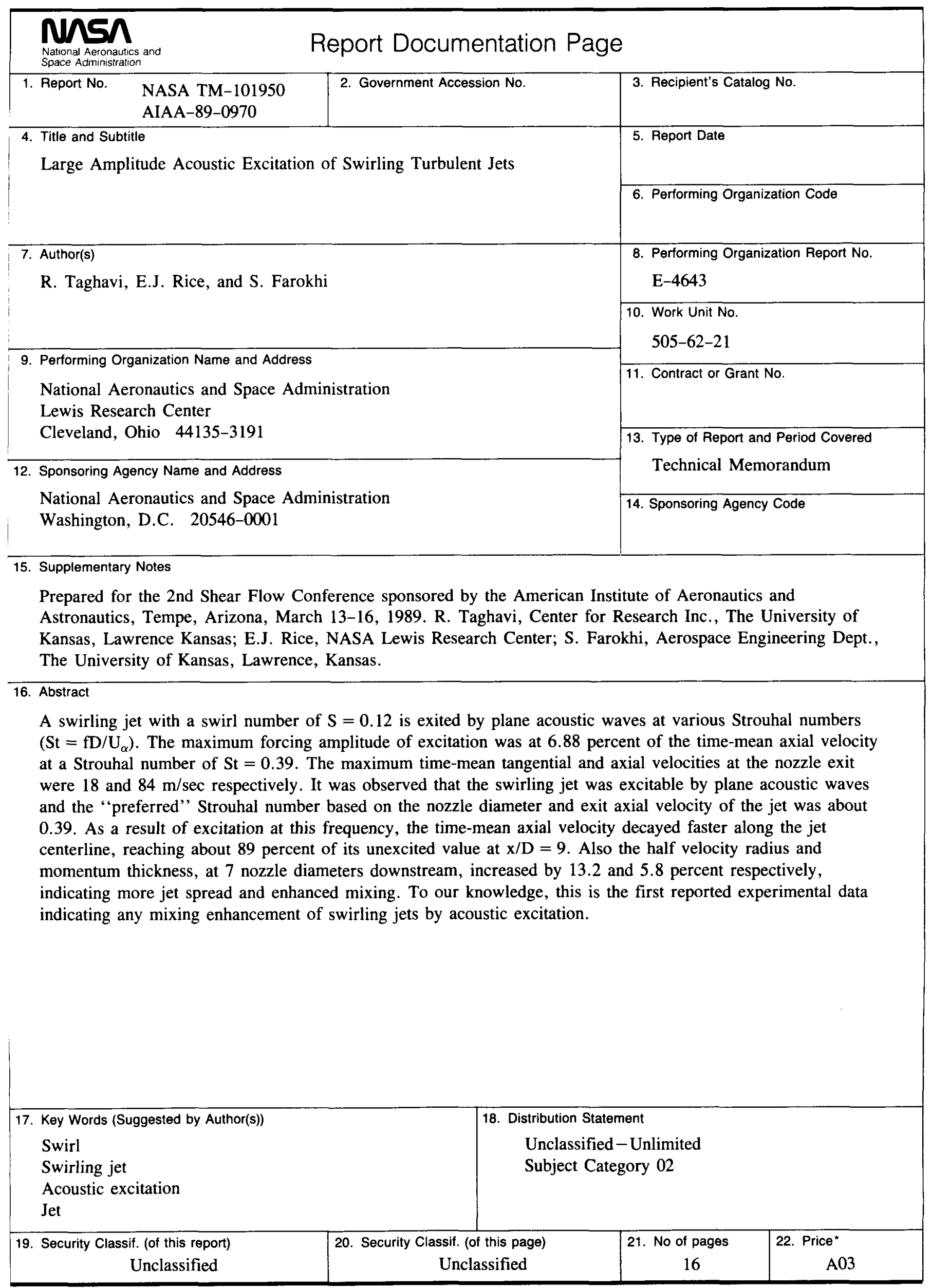

